\title{
Who's related to whom? Use published phylogenies and make customized tree-thinking assessments
}

\author{
Luke D. Blacquiere ${ }^{1}$, Allia Fawaz ${ }^{2}$ and William J. Hoese ${ }^{3^{*}}$ (D)
}

\begin{abstract}
A phylogeny depicts the hypothesized evolutionary relationships among taxa as a nested hierarchical branching diagram. Interpreting the relationships among taxa on a phylogeny is part of a set of skills called tree-thinking. Because published phylogenies are not constructed for the purpose of tree-thinking pedagogy, the information can be difficult for students to interpret and explicit instruction is required for mastery of the tree-thinking skill-set. We present a process to construct customizable assessment questions using published phylogenies, to assess a key tree-thinking skill, determining relatedness among taxa on a phylogeny. We detail how to construct two types of forced-choice questions: binary-choice and four-choice. In both question types, students are presented with a phylogeny and are instructed to determine which taxon from a list of taxa is most closely related to a focal taxon. The list of taxa includes distracters as possible responses explicitly selected based on common alternative strategies (similarity, proximity, node counting), in addition to the correct response. Instructors can select taxa of their own choosing in order to customize assessments. These assessment questions can be utilized during instruction as a formative assessment to enhance learning or in a summative assessment.
\end{abstract}

Keywords: Discipline-based (biology) education research, Evolution, Pedagogy, Evolutionary tree, Alternative strategies

\section{Introduction to evolutionary trees}

A phylogeny or evolutionary tree, is a graphical representation of the evolutionary history of life; it illustrates the hereditary connections between ancestors and descendants at any temporal scale (Avise 2006; Gregory 2008; Baum and Smith 2013; Dees et al. 2014, 2018). More specifically, an evolutionary tree is a nested hierarchical branching diagram that depicts hypothesized relationships among taxa, shows the outcomes of evolutionary processes, and illustrates how life is related by common ancestry. Evolutionary trees show hypothesized

\footnotetext{
${ }^{*}$ Correspondence: bhoese@fullerton.edu

${ }^{3}$ California State University, 800 N. State College Blvd, Fullerton, CA 92831, USA

Full list of author information is available at the end of the article
}

relationships among taxa and branch length does not represent time scale or genetic divergence. Evolutionary trees are essential tools for biologists (Kong et al. 2017) and learning to interpret information presented in an evolutionary tree is an important skill for introductory college-level biology students (Meir et al. 2007).

\section{Tree-thinking}

Tree-thinking includes a suite of skills and conceptual understanding that enables one to read and interpret evolutionary trees correctly (Baum et al. 2005; Thanukos 2009; Halverson 2011; Novick et al. 2011; Baum and Smith 2013; Novick and Catley 2013, 2016; Kummer et al. 2019; Schramm and Schmiemann 2019). College students often struggle when first learning to interpret the information in evolutionary trees (Baum et al. 2005; 
Catley 2006; Meir et al. 2007; Gregory 2008; Morabito et al. 2010; Halverson 2011; Novick and Catley 2013; Blacquiere and Hoese 2016; Dees et al. 2018; Kummer et al. 2019). These struggles may continue after initial instruction (Sandvik 2008; Halverson et al. 2011; Catley et al. 2012; Phillips et al. 2012; Dees et al. 2014), and impair student understanding of evolution and how it explains both the unity and diversity of life (Starr et al. 2012).

We developed a process for instructors to build customizable inductive curricular modules that can be used for teaching about and assessing understanding of relationships among taxa on a phylogeny, a key tree-thinking skill (Baum et al. 2005; Novick and Catley 2013). Phylogenies are common in scientific publications (Catley and Novick 2008; Kong et al. 2017) yet they are not constructed for the purpose of tree-thinking pedagogy therefore information in these published phylogenies can be difficult for students to extract and understand. The process we present demonstrates how to modify published phylogenies to use as teaching and assessment tools while retaining the fundamental information, evolutionary relationships among taxa, from the phylogenies. Other assessments for evaluating understanding of evolutionary relationships on phylogenies and additional tree-thinking skills (e.g., character mapping and determining the most parsimonious relationship among taxa) have been developed (Baum et al. 2005; Smith et al. 2013; Blacquiere and Hoese 2016). The process that we present is unique because it enables instructors to construct instructional modules and assessments using real data sets, the published phylogenies. These include questions that require students to evaluate evolutionary relationships among taxa on evolutionary trees with common incorrect strategies used as distracters to the correct response.

\section{Common incorrect strategies}

To demonstrate how to modify published phylogenies to construct questions that require students to evaluate evolutionary relationships among taxa on evolutionary trees, we show an initial phylogeny (Fig. 1a) that depicts the evolutionary relationships of the taxa as might be seen in a scientific publication (Catley and Novick 2008; Kong et al. 2017). Next, by rotating clades at internal nodes (Fig. 1b-e), a modification that does not change the relationships on the phylogeny, we illustrate how instructors can build assessment questions that include three common incorrect strategies: similarity, proximity, and node counting as distractors (Baum et al. 2005; Meir et al. 2007; Gregory 2008; Perry et al. 2008; Halverson et al. 2011; Novick and Catley 2013; Dees et al. 2014).
The similarity strategy (Fig. 1b) determines evolutionary relationships based on phenotypic similarity; the greater the phenotypic similarity between two taxa, the closer the evolutionary relationship between those two taxa. The fallacy of the similarity strategy is that closely related taxa always look like one another; however, this is not necessarily the case-more distantly related taxa may look similar to one another because of symplesiomorphies (shared ancestral characters) or homoplasies (independently derived characters). Therefore, students who use this approach answer questions incorrectly or answer questions correctly for the wrong underlying reason (Baum et al. 2005; Gregory 2008; Halverson et al. 2011; Novick and Catley 2013). Figure 1b shows an example of an assessment question that uses the similarity strategy as a distracter. The wolf is the focal taxon, the taxon to whom other taxa relationships, in this case the thylacine and the humpback whale, will be compared. Wolves and thylacines evolved similar body forms independently of one another, an example of homoplasic characters. Although wolves are evolutionarily more closely related to humpback whales than thylacines, students with the false impression that this body form indicates relatedness would incorrectly assume that the wolf is more closely related to the thylacine than the humpback whale.

The common incorrect strategy proximity (Fig. 1c) assumes that the distance between taxa along the branch tips is inversely proportional to relatedness; as the distance between taxa along the branch tips decreases, how closely the taxa are related increases (Baum et al. 2005). Figure 1c shows a question with the proximity strategy as a distracter. Although orangutans are evolutionarily more closely related to zebras than thylacines, students who use the proximity strategy erroneously conclude the orangutan to be more closely related to the thylacine because they are closer to one another along the branch tips than the orangutan is to the zebra along the branch tips.

In the node counting strategy (Fig. 1d), the number of internal nodes separating taxa is used to determine relatedness; taxa with fewer intervening nodes separating them are considered to be more closely related to one another than taxa with a greater number of intervening nodes separating them (Baum et al. 2005; Meir et al. 2007; Gregory 2008; Perry et al. 2008; Halverson et al. 2011; Dees et al. 2014). This strategy stems from the faulty notion that evolutionary change only occurs at the nodes, with each node indicating a single change (Baum et al. 2005; Gregory 2008). In Fig. 1d, the node counting strategy is a distracter response. Although wolves are evolutionarily more closely related to 


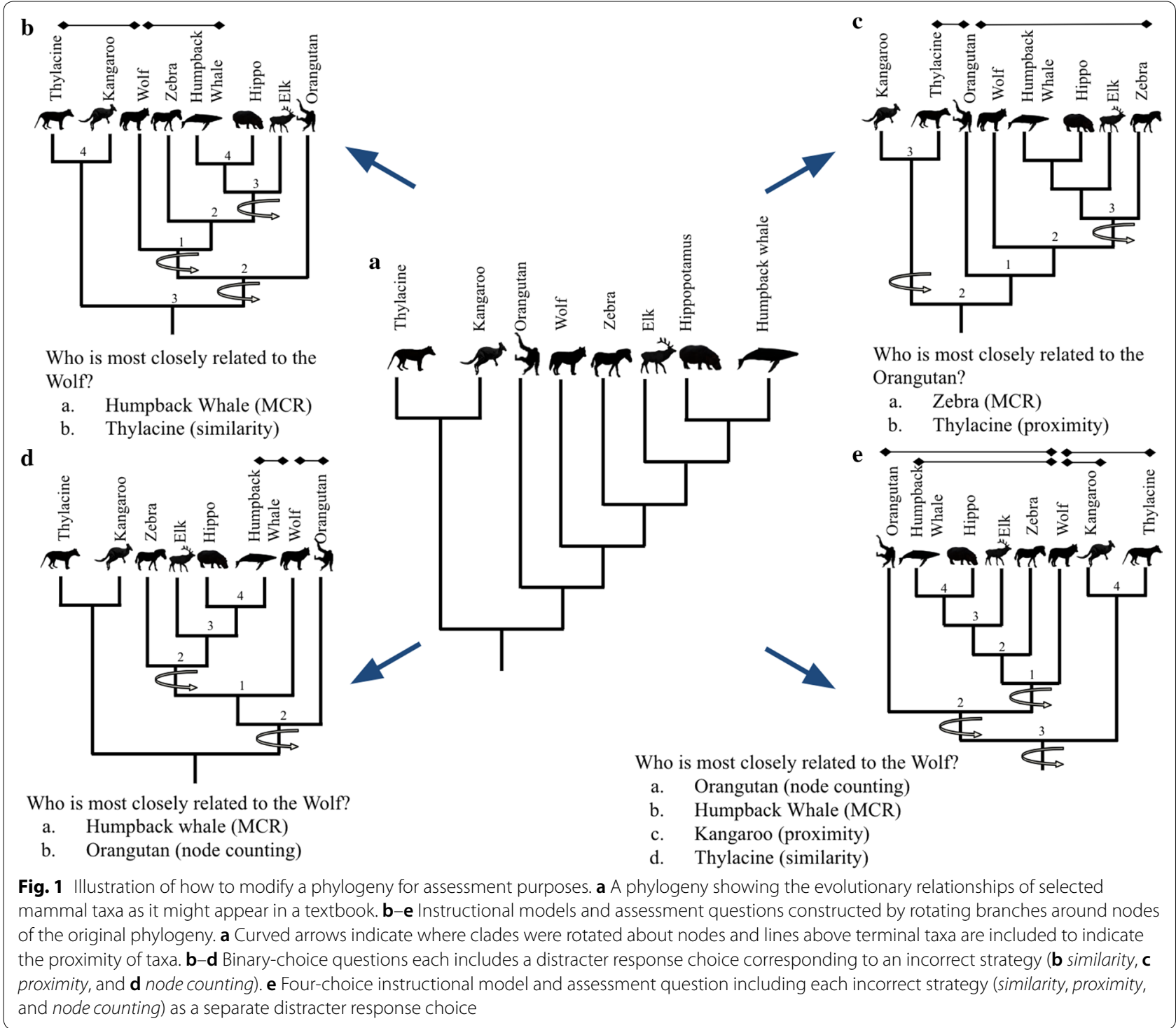

humpback whales than orangutans, a student using the node counting strategy would conclude that the orangutan is more closely related to the wolf than the humpback whale because two internal nodes separate the wolf and the orangutan whereas four internal nodes separate the wolf and humpback whale.

Evolutionary trees can also be structured so multiple different distracters can be embedded in a single, multiple choice question, each response using a single distracter (Fig. 1e). Although wolves are more closely related to humpback whales than the other response choices, a student using the similarity strategy would be distracted by the thylacine response, a student using the proximity strategy would be distracted by the kangaroo response, and a student using the node counting strategy would be distracted by the orangutan response (Fig. 1e).

\section{An overview of question building to address common incorrect strategies}

We describe a method in which instructors can use taxa of their choosing to build their own instructional modules and assessment questions focused on the use of a most recent common ancestor to determine evolutionary relatedness: a tree-thinking skill that is fundamental for understanding how to interpret the information in evolutionary trees. Next, we describe how we used the 15 possible different rooted trees for four taxa to construct assessment trees that use three common incorrect approaches (i.e., similarity, proximity, and node counting) 
as distracters. We then describe how to use information in published phylogenies, along with the rooted trees and corresponding assessment trees, to build custom assessment questions. We provide several examples of the question-building process to aid instructors in constructing their own questions using taxa of their choosing. In addition, the examples provided may also serve as readymade sample questions for instructors.

\section{Question development procedure}

We developed two categories of forced-choice questions that can be used to assess understanding of relationships among taxa in an evolutionary tree. Each question includes an evolutionary tree, which students will reference to determine their response. Evolutionary trees can be presented in a variety of formats (i.e., rectangular, diagonal, or circular); we present evolutionary trees in rectangle format because students exhibit greater accuracy and sophisticated reasoning when interpreting relationships among taxa on evolutionary trees in this format (Novick and Catley 2007, 2013). The evolutionary trees presented in questions are derived from assessment trees (Figs. 2, 4), which are a key piece of the customization process. Assessment trees differ from evolutionary trees because they do not include taxa along the branch tips; instead, they indicate where to place the focal, most closely related, and distracter taxa that will be included in the question narrative and include letters that represent taxa that are not part of the question narrative. Because the roles of the taxa (e.g., focal, similarity, proximity), but not the taxa themselves, are included on assessment trees, questions can be customized by instructors who determine which taxa to use to fill the roles. In each question, students are prompted to identify which taxon, from a list of choices, is most closely related to a focal taxon. In both categories, the correct response is the taxon that shares a more recent common ancestor with the focal taxon when compared to other taxon responses. The

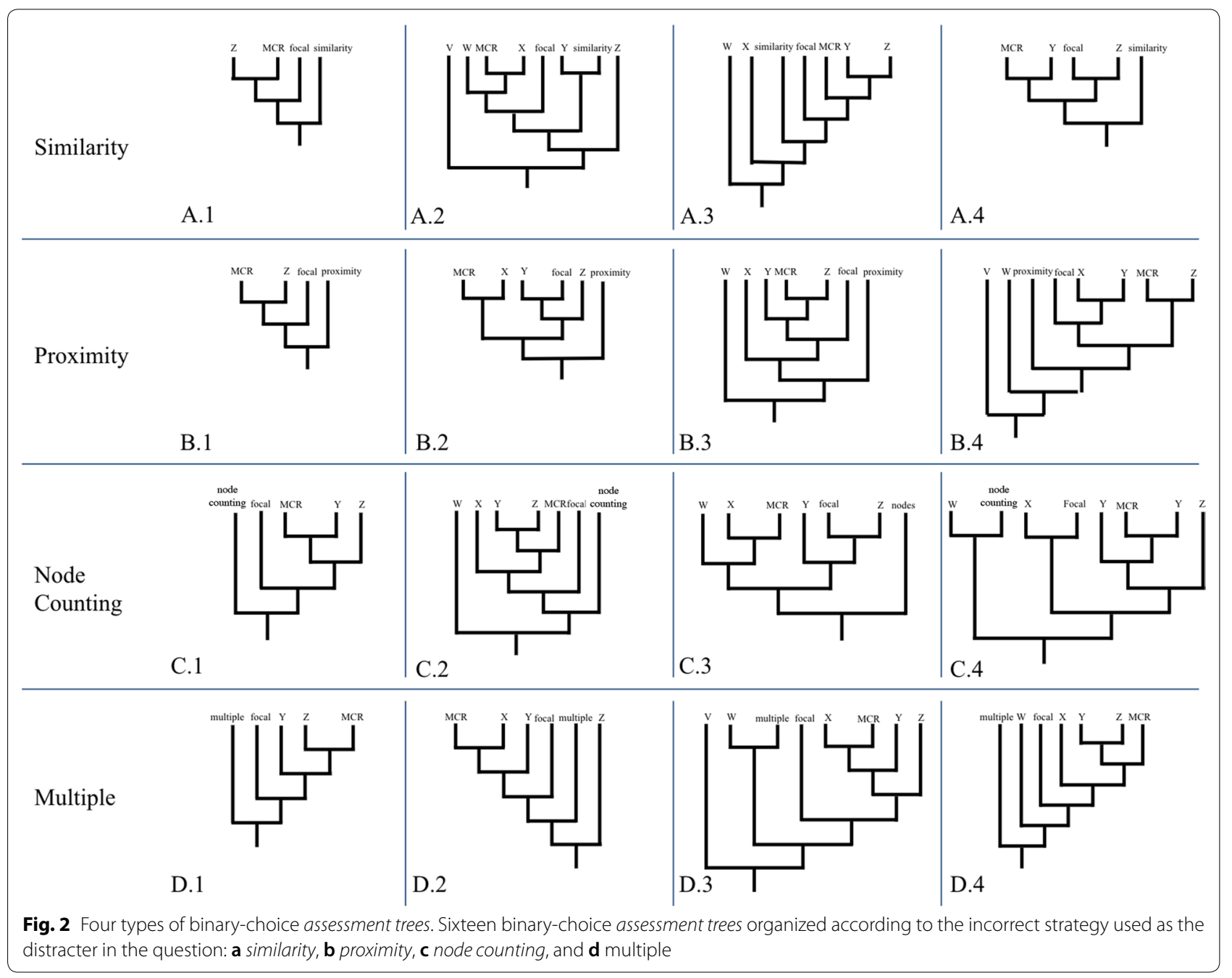


correct response, the most closely related (MCR) taxon, has greater phenotypic differences with the focal taxon than the similarity distracter taxon, has a greater distance separating it from the focal taxon along the branch tips than the proximity distracter taxon, and has more intervening nodes between it and the focal taxon than the node counting distracter taxon.

\section{Binary-choice assessment trees}

The first category of questions are binary-choice; the student determines which of two taxa, the distracter or the MCR, is more closely related to a focal taxon. We developed four different types of questions in this category. The distracter response choice in each type of question represents one or all three distracters: similarity, proximity, node counting, and multiple. Four example assessment trees are provided for each type of question. Question types are referenced by the distracter or distracters (in the case of multiple) that are included as a response choice. The first assessment tree example (Fig. 2) for each question type has the fewest number of lineages. The remaining three assessment tree examples included in each question type retain the evolutionary relationship of the focal, MCR, and distracter taxa, while lineages

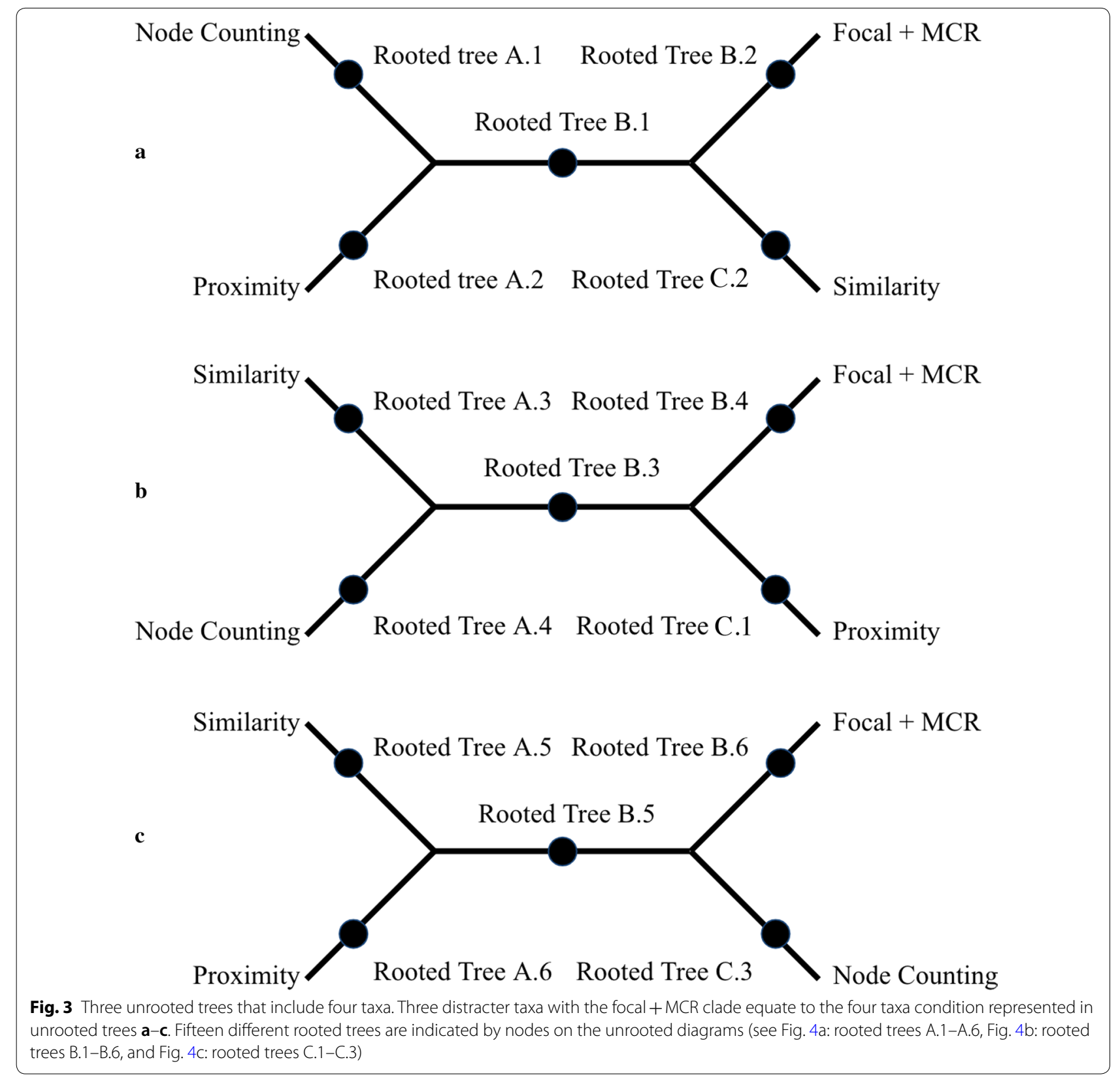




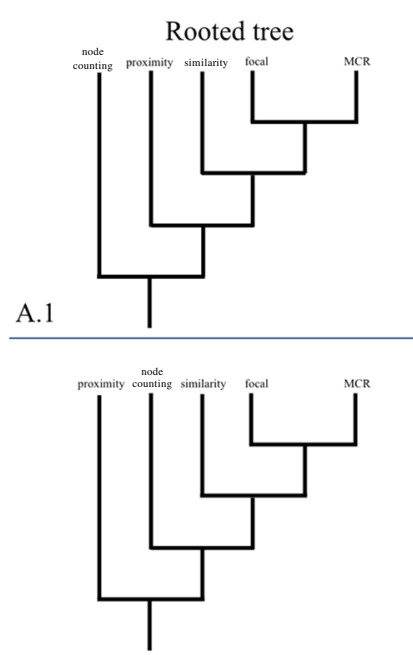

A. 2

A. 3

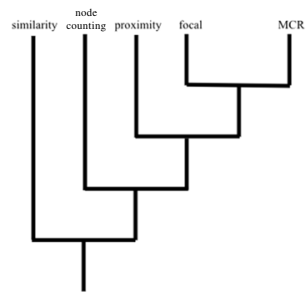

A. 4

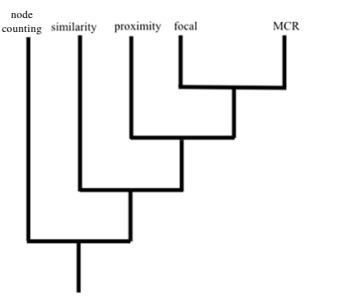

A. 5
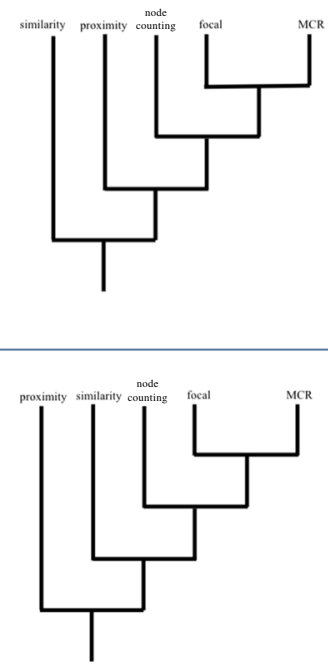

Assessment tree 1

Assessment tree 2
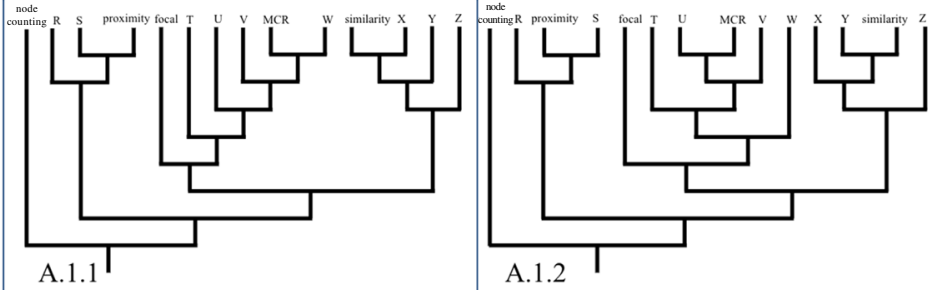

A.1.2

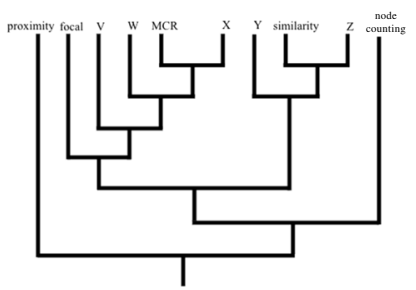

A.2.1

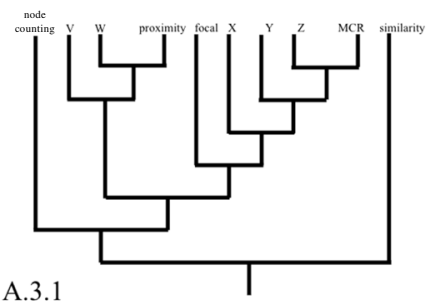

A.3.2

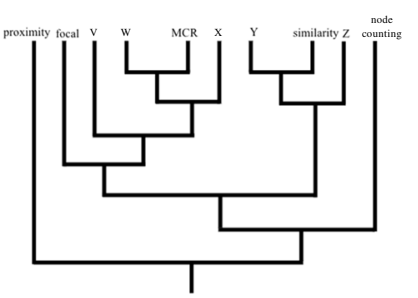

A.2.2
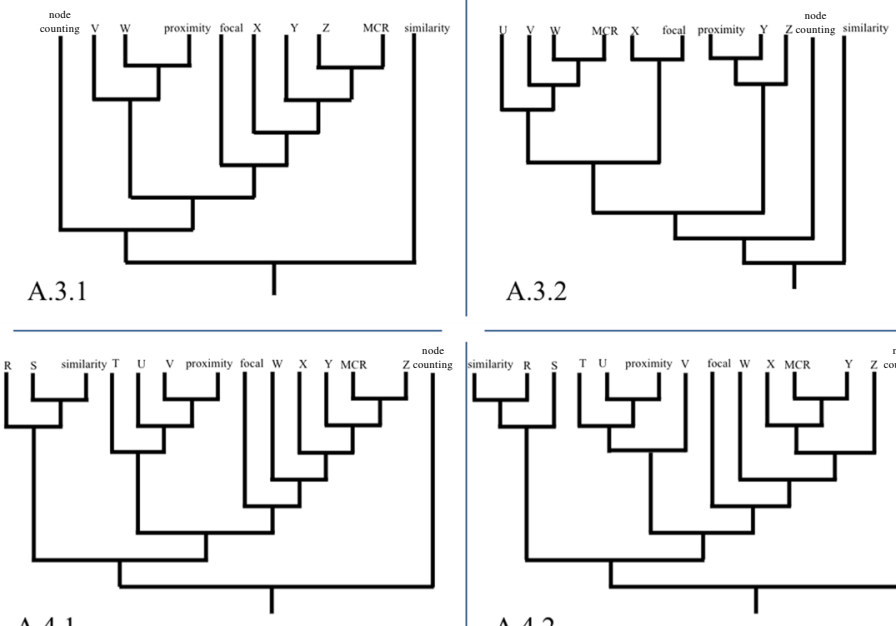

A.4.1

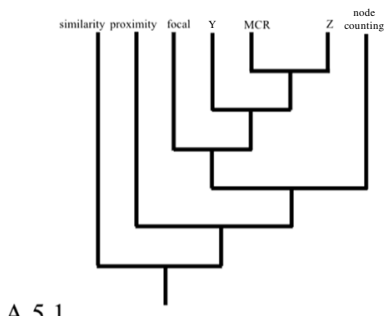

A. 5.1

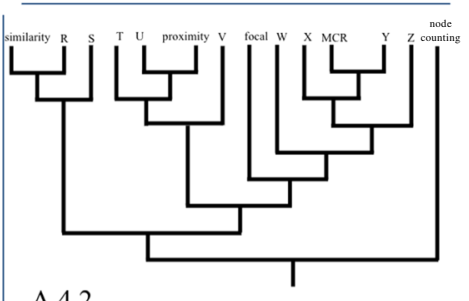

A.4.2

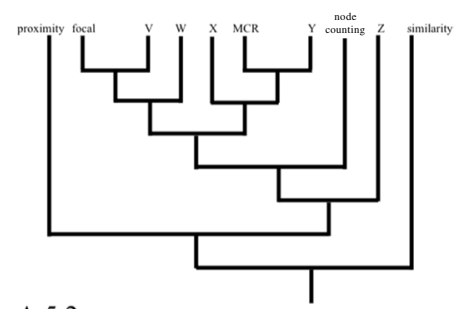

A.5.2
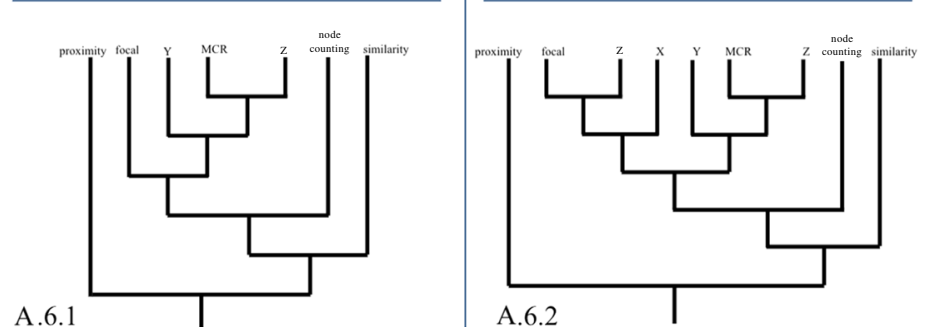

Fig. 4 Four-taxa rooted trees with corresponding assessment trees. Fifteen possible four-taxa rooted trees with thirty corresponding assessment trees. The left column includes all fifteen possible four-taxa rooted trees organized by branching pattern a (A.1-A.6), b (B.1-B.6), and $\mathbf{c}$ (C.1-C.3). The middle and right columns include all thirty assessment trees corresponding to each of the fifteen rooted trees 


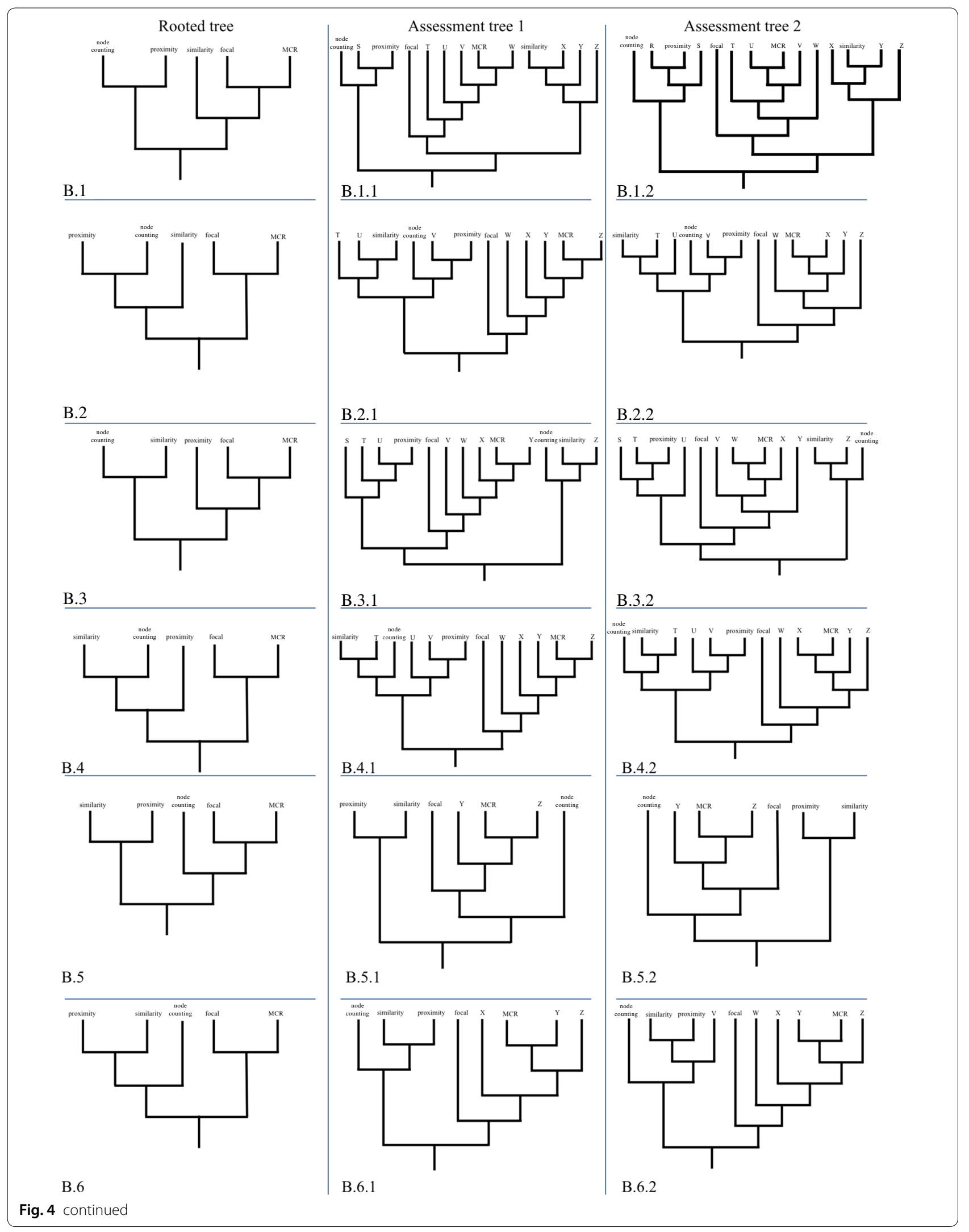




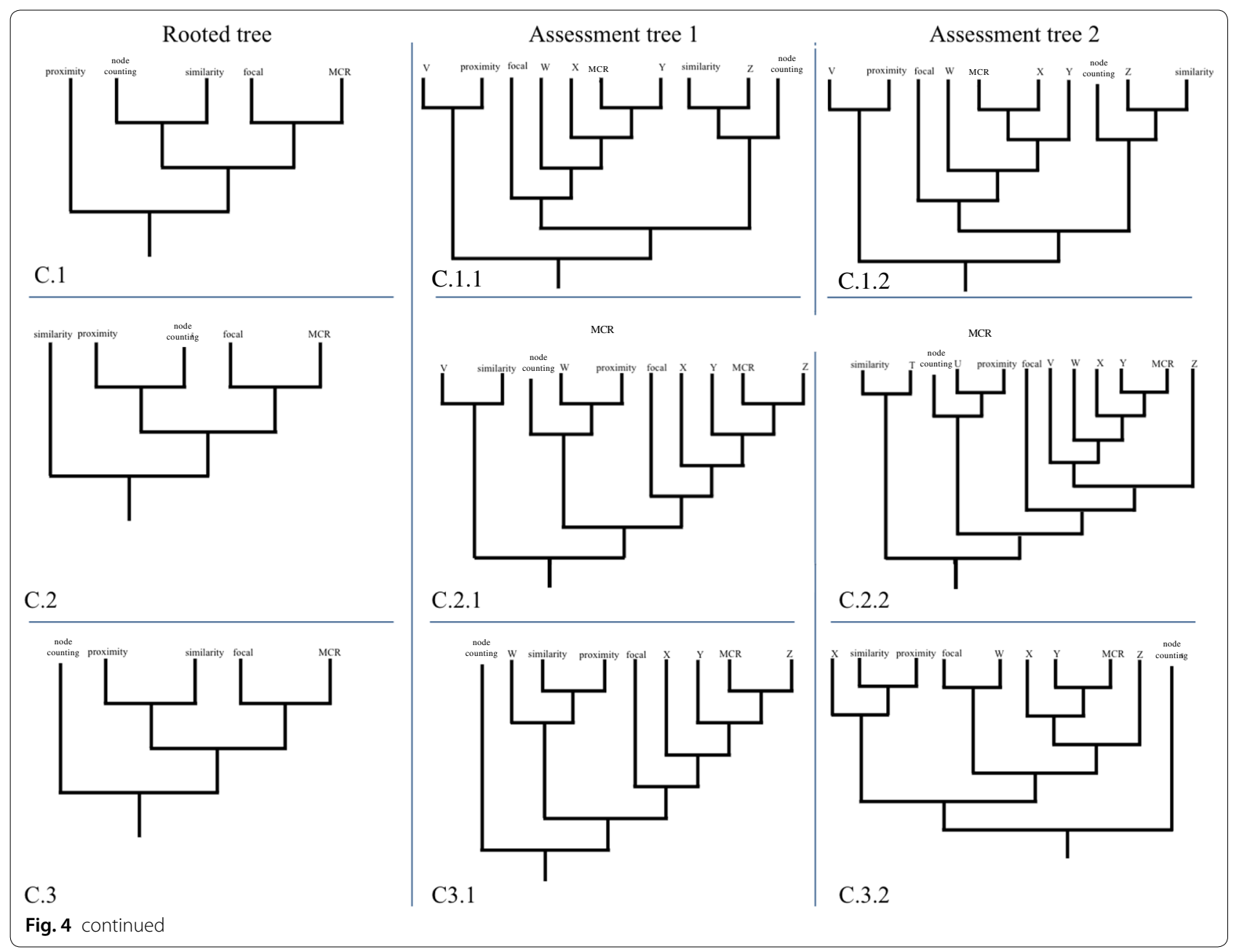

have been added and clades have been rotated at nodes in order to vary the appearance of the evolutionary trees. This was done to make it less likely that students memorize the correct response for a particular evolutionary tree. The sample trees can also be mirrored, which increases the number of different looks in questions, further reducing the chance that students could memorize a correct answer following an example.

Questions constructed to exhibit one incorrect strategy as a distracter control for the use of the other two incorrect strategies in the answer selections. For example, assessment trees in the similarity question type present two taxa response choices that have equal proximity to the focal taxon along the branch tips and are separated from the focal taxon by the same number of internal nodes. Thus, these other strategies do not provide cues to help students decide which response choice is correct. When answering the question, students decide between two response choices: the MCR taxon, which is more closely related to the focal taxon than the other response

\section{(See figure on next page.)}

Fig. 5 Question development using published phylogeny of vertebrate taxa, assessment trees, and images. a Taxa selected from a vertebrate phylogeny (modified from Fig. 1 in Meyer and Zardoya 2003) with selected clades color-coded. b Assessment categories for each taxonomic group. c Taxa selected for the assessment question: crocodile (focal; green), bird (MCR; green), lizard (similarity; yellow), humpback whale (proximity; blue), and bat (node counting; blue). d Color-coded rooted tree, B.1 (Figs. 3, 4b), including an image and the role of each taxon. e Assessment tree developed with tree B.1.1 (Fig. 4b). The assessment tree is configured by branch addition and clade rotation about nodes so taxa acting as distracters in the question represent their respective incorrect strategy. Sample question: Who is most closely related to the Crocodile? a. Bat, b. Humpback Whale, c. Bird, d. Lizard 

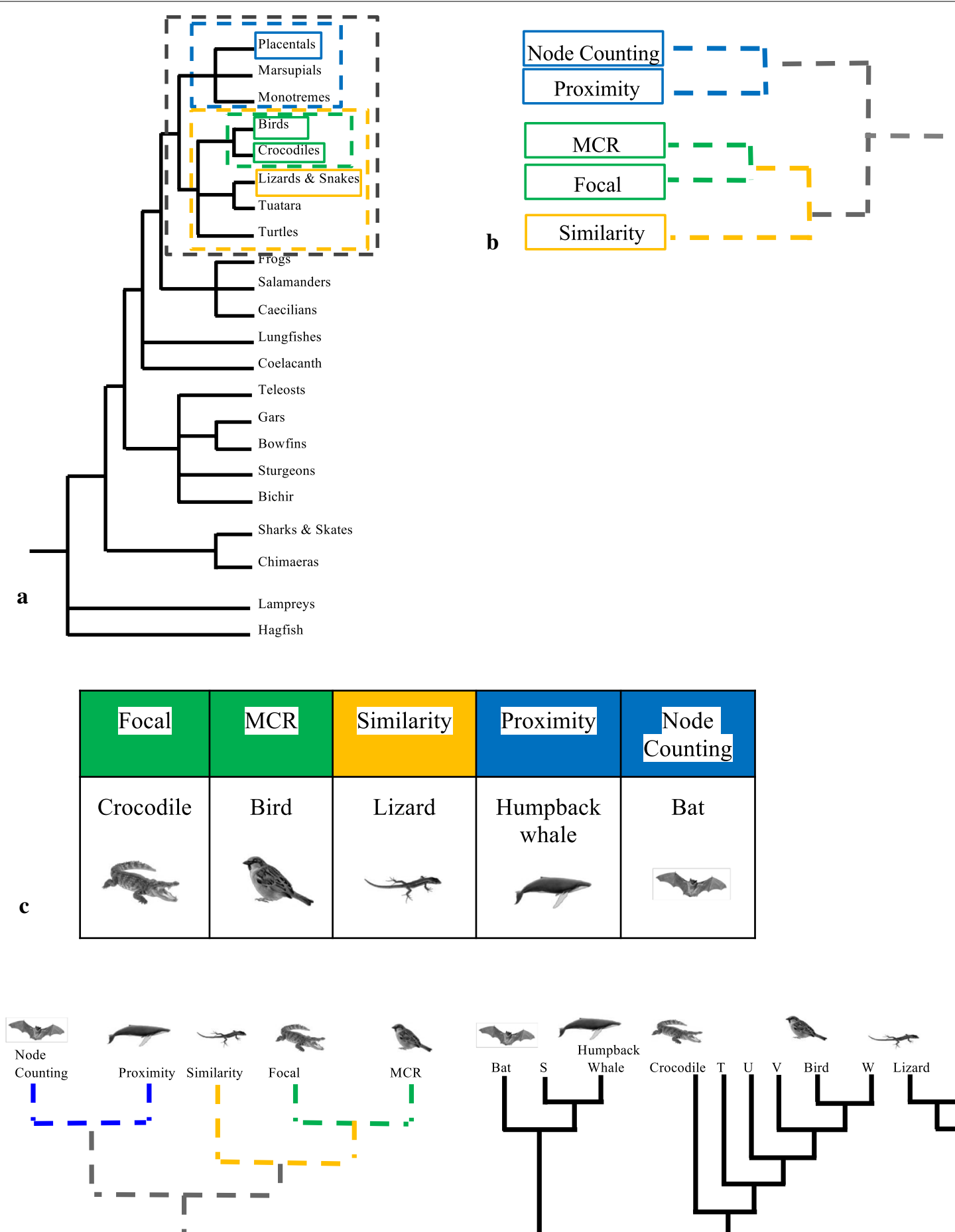

d Rooted Tree: B.1

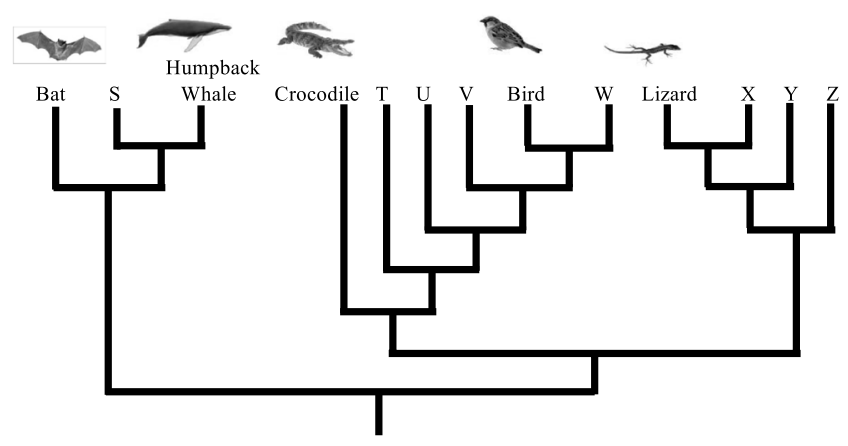

e Question based on Assessment Tree: B.1.1 
(See figure on next page.)

Fig. 6 Question development using published phylogeny of vertebrate taxa, assessment trees, and images. a Taxa selected from a vertebrate phylogeny (modified from Fig. 1 in Meyer and Zardoya 2003) with selected clades color-coded. b Assessment categories for each taxonomic group. c Taxa selected for the assessment question: coelacanth (focal; green), frog (MCR; green), grouper (similarity; purple), ray (proximity; blue), and lamprey (node counting; yellow). d A color-coded rooted tree, A.1 (Figs. 3, 4a). e Assessment tree developed with tree A.1.1 (Fig. 4a). The assessment tree is configured by branch addition and clade rotation about nodes so taxa acting as distracters in the question represent their respective incorrect strategy. Sample question: Who is most closely related to the Coelacanth? a. Lamprey, b. Ray, c. Frog, d. Grouper

choice, and the similarity taxon, which has greater phenotypic similarity to the focal taxon than the MCR taxon (Fig. 2, A.1-A.4).

In assessment trees with the proximity alternative strategy included as a distracter, participants are provided with two response choices with commensurate phenotypic similarity to the focal taxon (as pre-determined by the instructor) and both are separated from the focal taxon by the same number of internal nodes. One of the response choices, the MCR taxon, is more closely related to the focal taxon and the other response choice, the proximity taxon, is closer in proximity to the focal taxon along the branch tips than the MCR taxon (Fig. 2, B.1-B.4).

When node counting is the alternative strategy used as a distracter, participants are presented with two response choices that both have equal proximity to the focal taxon along branch tips and both are equal in phenotypic similarity to the focal taxon. One response choice, the MCR taxon, is more closely related to the focal taxon and the other response choice, the node counting taxon, is separated by fewer internal nodes from the focal taxon than the MCR taxon is from the focal taxon (Fig. 2, C.1-C.4).

The multiple question type includes a multiple-distracter response choice, which exhibits all three incorrect strategies described here, and the MCR taxon response choice. The multiple-distracter response choice is closer in phenotypic similarity to the focal taxon, closer in proximity along the branch tips to the focal taxon, and is separated from the focal taxon by fewer internal nodes than the MCR taxon (Fig. 2, D.1-D.4). This design results in students responding to the question incorrectly if they use any one or all of the three incorrect strategies when interpreting the evolutionary tree.

\section{Four-choice assessment trees}

In the second category of questions, students select from four response choices, one MCR taxon response choice and three distracter taxa response choices, when determining which taxon is most closely related to the focal taxon. Each of the three distracter response choices corresponds to one common incorrect strategy (similarity, proximity, or node counting). In total, five defined taxa will be included in the assessment trees for this category of question: focal, MCR, similarity, proximity, and node counting; other undefined taxa included on the assessment trees will be represented by letters.

The focal and the MCR taxa must share a more recent common ancestor with one another than either share with the distracter taxa; therefore, the focal and MCR taxa are always in a clade that does not include the distracter taxa and are treated as one taxonomic unit in the rooted and unrooted trees developed to construct assessment trees for these types of questions (see Fig. 3). Because the focal and MCR are treated as a single taxonomic unit, the construction of assessment trees for these questions essentially considers the relationship among four taxa. Three unrooted trees (Fig. 3a-c) illustrate all the different ways in which four taxonomic units can be arranged. The focal taxon and response choice taxa (MCR, proximity, similarity, and node counting) can be distributed in different permutations along the branch tips of the evolutionary tree depending on where each unrooted tree is rooted. Five rooted trees can be derived from each unrooted tree in Fig. 3. Each rooted tree is different because the relationships among the taxa are different (Fig. 4). On a rooted evolutionary tree with bifurcating branches, four taxonomic units can be related to one another in fifteen different ways (Balding et al. 2008). Rooted trees are not appropriate for use as assessments because they have not yet been manipulated to incorporate the features of the distracters.

We present 30 assessment trees (see Fig. 4); two example assessment trees were developed for each of the 15 rooted trees (e.g., assessment trees A.1.1 and A.1.2 are derived from rooted tree A.1; Fig. 4a). These assessment trees maintain the evolutionary relationships among the taxa from their corresponding rooted tree (e.g., the 

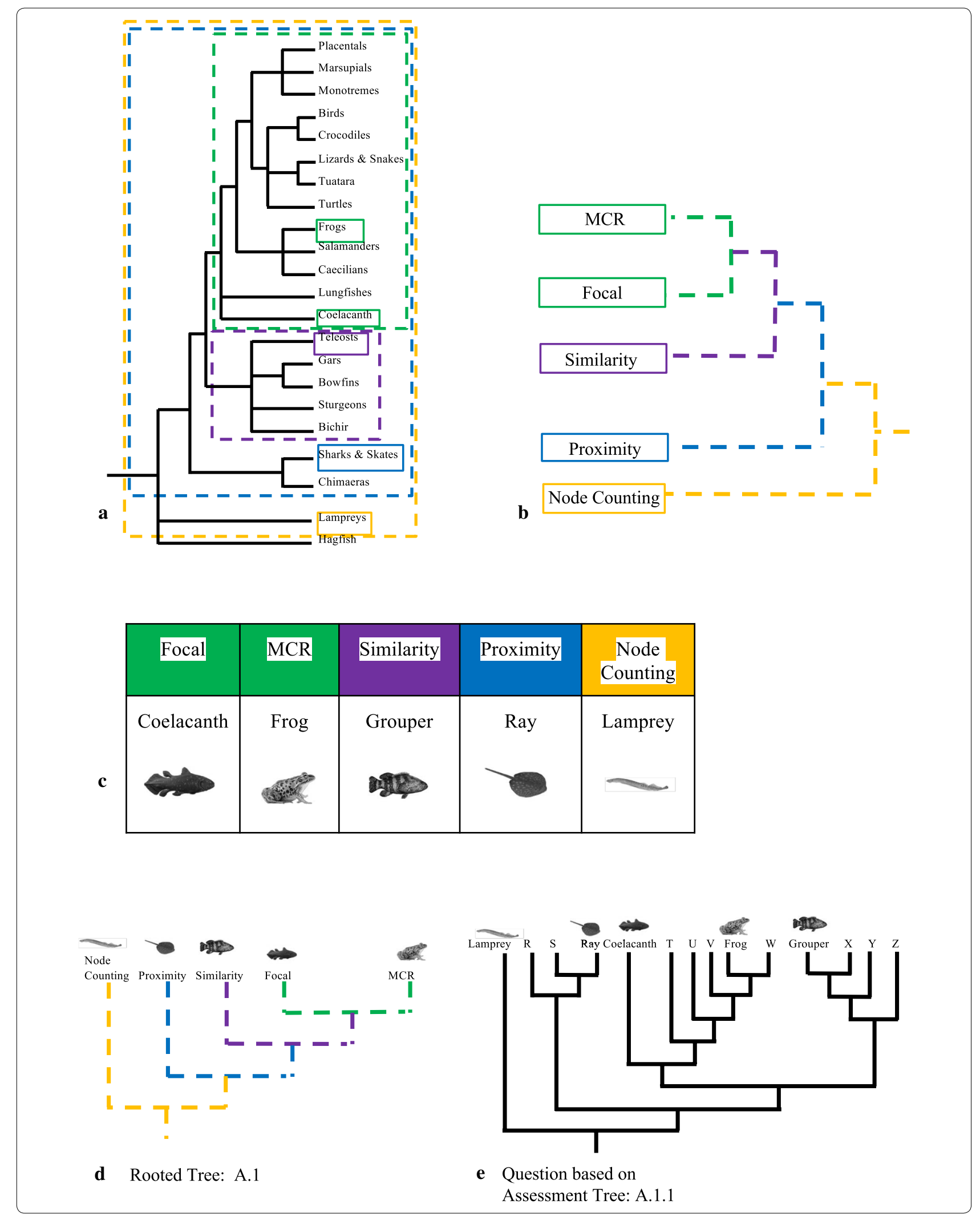


\section{(See figure on next page.)}

Fig. 7 Question development using published phylogeny of mammals, assessment trees, and images. a Taxa selected from a mammalian phylogeny (modified from Fig. 1 in Meredith et al. 2011) with clades color-coded. b Assessment categories for each taxonomic group. c Taxa selected for the assessment question: flying squirrel (focal; green), rabbit (MCR; green), flying phalanger (similarity; purple), cat (proximity; blue), and wolf (node counting; blue). d A color-coded rooted tree (C.2 in Figs. 3, 4c) is constructed with the selected taxa. e Assessment tree developed with tree C.2.1 (Fig. 4c). The assessment tree is configured by branch addition and clade rotation about nodes so taxa acting as distracters in the question represent their respective incorrect strategy. Sample question: Who is most closely related to the Flying Squirrel? a. Flying Phalanger, b. Wolf, c. Cat, d. Rabbit

evolutionary relationships among taxa on rooted tree A.1 is maintained on both assessment trees A.1.1 and A.1.2 in Fig. 4a) but they have been modified so that each alternative strategy distracter response choice is controlled for the other two incorrect strategy distracter response choices to an extent that they do not provide cues to help students decide which response choice is correct.

Assessment trees developed for the four-choice questions are similar to the assessment trees in the binarychoice questions in that they indicate where to place the five taxa included in the question narrative and show letters that represent taxa not included in the question narrative. To develop assessment trees for the four-choice questions, which presents distracters that control for the use of the other incorrect strategies, modifications were made to the structure of the rooted tree while maintaining the evolutionary relationships among taxa on the rooted tree. The modifications include adding or removing branches (lineages) and rotating clades at internal nodes. The assessment trees are designed so that the similarity taxon is more phenotypically similar to the focal taxon than the other taxa response choices, the proximity taxon is closer in proximity to the focal taxon along the branch tips than the other taxa response choices, and the node counting taxon is separated by fewer internal nodes from the focal taxon than the other taxa response choices.

\section{Images of taxa}

Images were selected to accompany the taxa that are included in the question narrative. The images are included because the use of the similarity alternative strategy requires students to compare the phenotypic similarity of the taxa included in the question narrative. The primary rule for selecting images is the image of the focal taxon and the similarity taxon must appear more phenotypically similar to each other than either does to the other taxon in binary-choice or taxa in four-choice questions.

We suggest: (1) selecting images without a background to reduce distraction and make the subject clearer and (2) maintaining a consistent angle of view (we typically used a lateral or dorsal view). After the taxa that are to be included on the assessment tree have been selected, Microsoft Office picture formatting can be used to change the color to grayscale to eliminate cues that may act as lurking variables. Other software programs can be used for image manipulation. We present an abbreviated procedure using Microsoft ${ }^{\circledR}$ Word 2019 version 16.29.1 because it is widely used and available.

Procedure for modifying images using Microsoft Office:

1. Insert image into Microsoft Office document.

2. Select the image.

3. Select picture format tab.

4. Select the Color option drop-down menu.

5. In the Recolor menu select grayscale.

6. Copy and paste in the appropriate location on the assessment tree.

7. Resize as necessary, maintaining aspect ratio.

\section{Creating your own assessment questions}

Assessment trees enable instructors to use taxa from any phylogeny they choose to construct pedagogically designed questions that can be used for instruction or assessment. Two types of questions, binary-choice and four-choice, can be developed using the resources provided. Given the four assessment trees for each distracter to be used with binary-choice questions and two assessment trees for each of the fifteen phylogenies that depict relationships among five taxa (focal, MCR, similarity, 
a
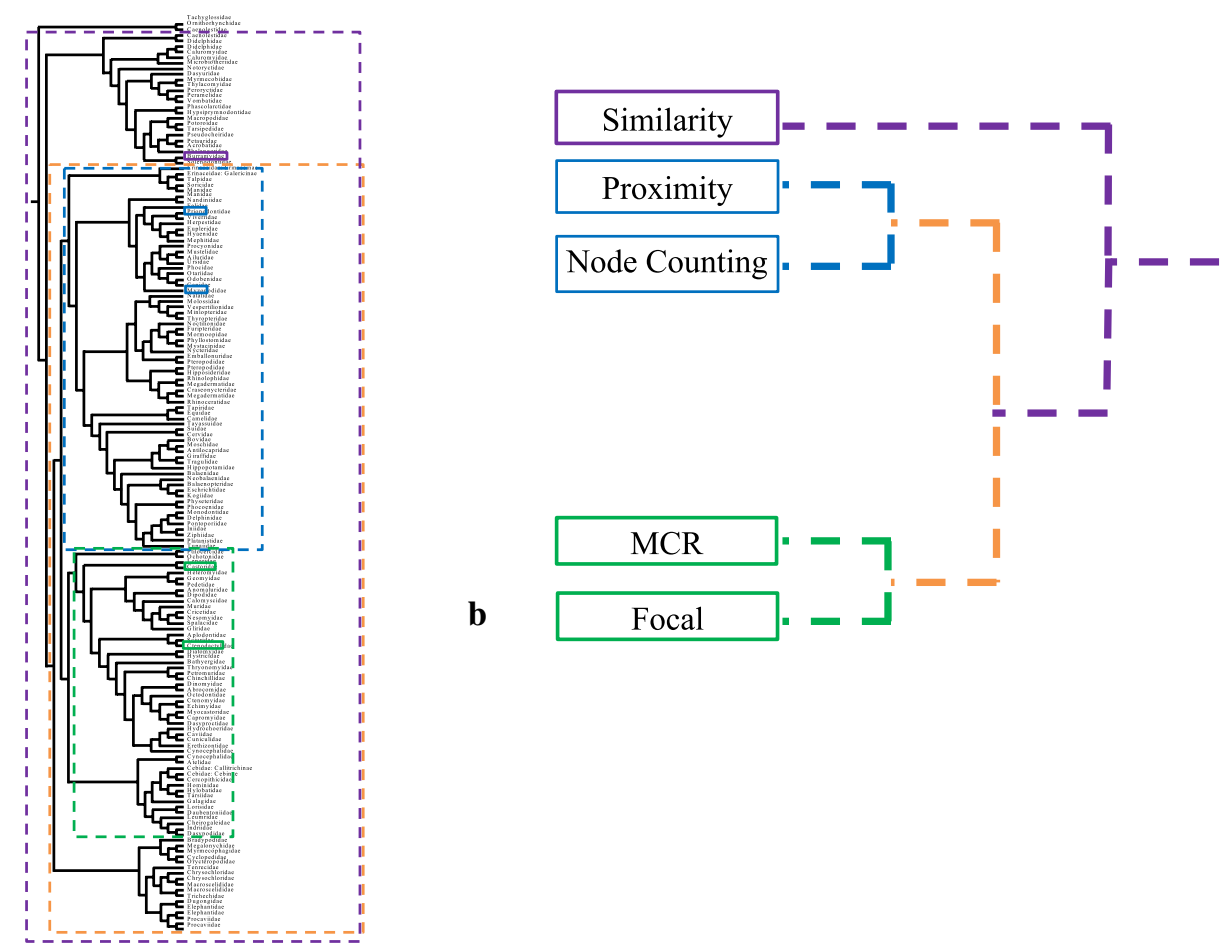

\begin{tabular}{|c|c|c|c|c|}
\hline Focal & MCR & Similarity & Proximity & $\begin{array}{c}\text { Node } \\
\text { Counting }\end{array}$ \\
\hline $\begin{array}{l}\text { Flying } \\
\text { Squirrel }\end{array}$ & Rabbit & $\begin{array}{l}\text { Flying } \\
\text { Phalanger }\end{array}$ & Cat & Wolf \\
\hline 2 & & & & $\sqrt{1}$ \\
\hline
\end{tabular}
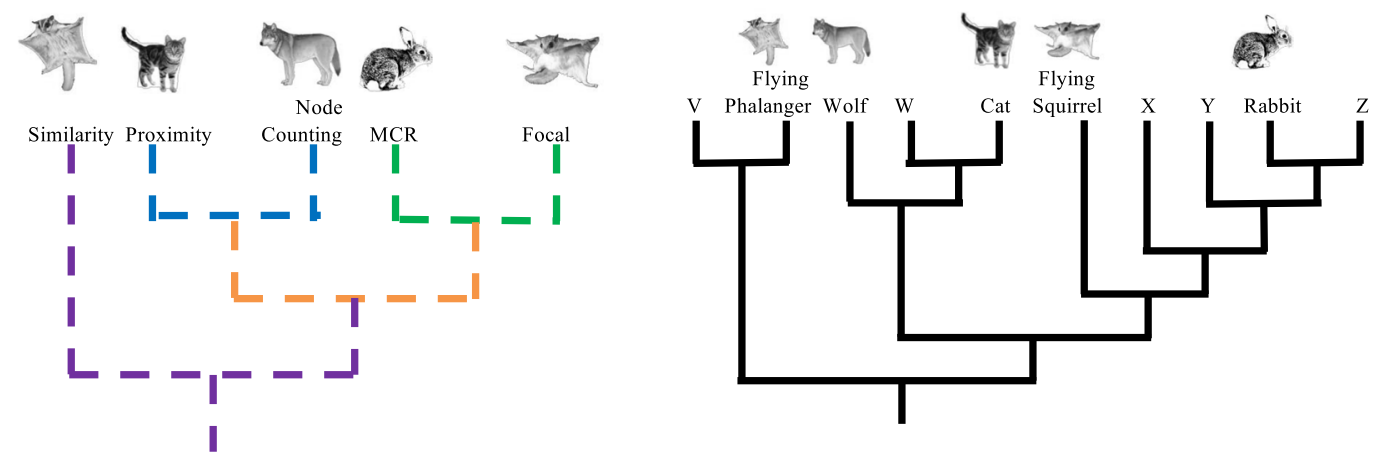

d Rooted tree: C.2

e Question Based on

Assessment Tree: C.2.1 


\section{(See figure on next page.)}

Fig. 8 Question development using published phylogeny of mammals, assessment trees, and images. a Taxa selected from a mammalian phylogeny (modified from Fig. 1 in Meredith et al. 2011) with clades color-coded. b Assessment categories for each taxonomic group. c Taxa selected for the assessment question: deer mouse (focal; green), tiger (MCR; green), Antechinus (similarity; yellow), kangaroo (proximity; purple), and wombat (node counting; purple). d A color-coded rooted tree, B.2 (Figs. 3, 4b). e Assessment tree B.2.1 (Fig. 4b). The assessment tree is configured by branch addition and clade rotation about nodes so taxa acting as distracters in the question represent their respective incorrect strategy. Sample question: Who is most closely related to the Deer Mouse? a. Antichinus, b. Wombat, c. Kangaroo, d. Tiger

proximity, and node counting) for four-choice questions, instructors have a wide variety of tree structures and taxonomic choices in order to develop their own questions. Separate assessments were constructed each containing one question type, binary-choice or four-choice, and were analyzed for discrimination, validity, and reliability (Fawaz 2015; Blacquiere and Hoese 2016). Both assessment types were valid, exhibited high reliability, and discriminated between students using the correct strategy and those using an incorrect strategy to determine evolutionary relationships among taxa. We provide the processes to construct binary-choice and four-choice questions with several example questions. All trees include images of the response choices; the phenotypic similarity distracter requires students to view and compare images based on this similarity.

\section{Creating custom binary-choice questions}

Use the following procedure to construct a binary-choice question:

1. Select a published phylogeny, or one of our phylogenies developed from a published phylogeny, that includes the three taxa (focal, MCR, distracter) that will be included in the question and identify the type of question you want to develop (similarity, proximity, node counting, or multiple).

2. Select the focal and MCR taxa from the phylogeny. The MCR taxon will share a more recent common ancestor with the focal taxon than with the distracter taxon.

3. Identify the distracter taxon. If the question contains node counting or proximity, select the node counting or proximity taxon. For a question containing a similarity or multiple-distracter, the distracter taxon must be more phenotypically similar to the focal taxon than the MCR, as determined by the instructor.
4. Select and modify taxa images for the focal, MCR, and distracter taxon as described above.

5. Place the taxa and taxa images in the indicated places along the branch tips of one of the assessment trees in Fig. 2.

6. Write the question following the format below, substituting in the taxa names:

7. Which species is most closely related to the Focal taxon?
a. MCR taxon
b. Distracter taxon.

\section{Creating custom four-choice questions}

Use the following procedure to construct a four-choice question:

1. Select a published phylogeny or one of the phylogenies that includes the five taxa that will be included in the question (focal, MCR, similarity, proximity, and node counting).

2. Select the focal and MCR taxa from the phylogeny. The MCR taxon will share a more recent common ancestor with the focal taxon than with the distracter taxa.

3. Identify the similarity taxon. The similarity taxon must be more phenotypically similar to the focal taxon, as determined by the instructor, than the other taxa in the question (MCR, proximity, and node counting).

4. Select the proximity and node counting taxa.

5. Use the phylogeny selected in step 1 to determine which rooted tree and assessment tree from Fig. 4a (A.1-A.6), Fig. 4b (B.1-B.6), and Fig. 4c (C.1-C.3) depicts the evolutionary relationships of the five taxa.

6. Select and modify taxa images for the focal, MCR, and distracter taxa as described above. 


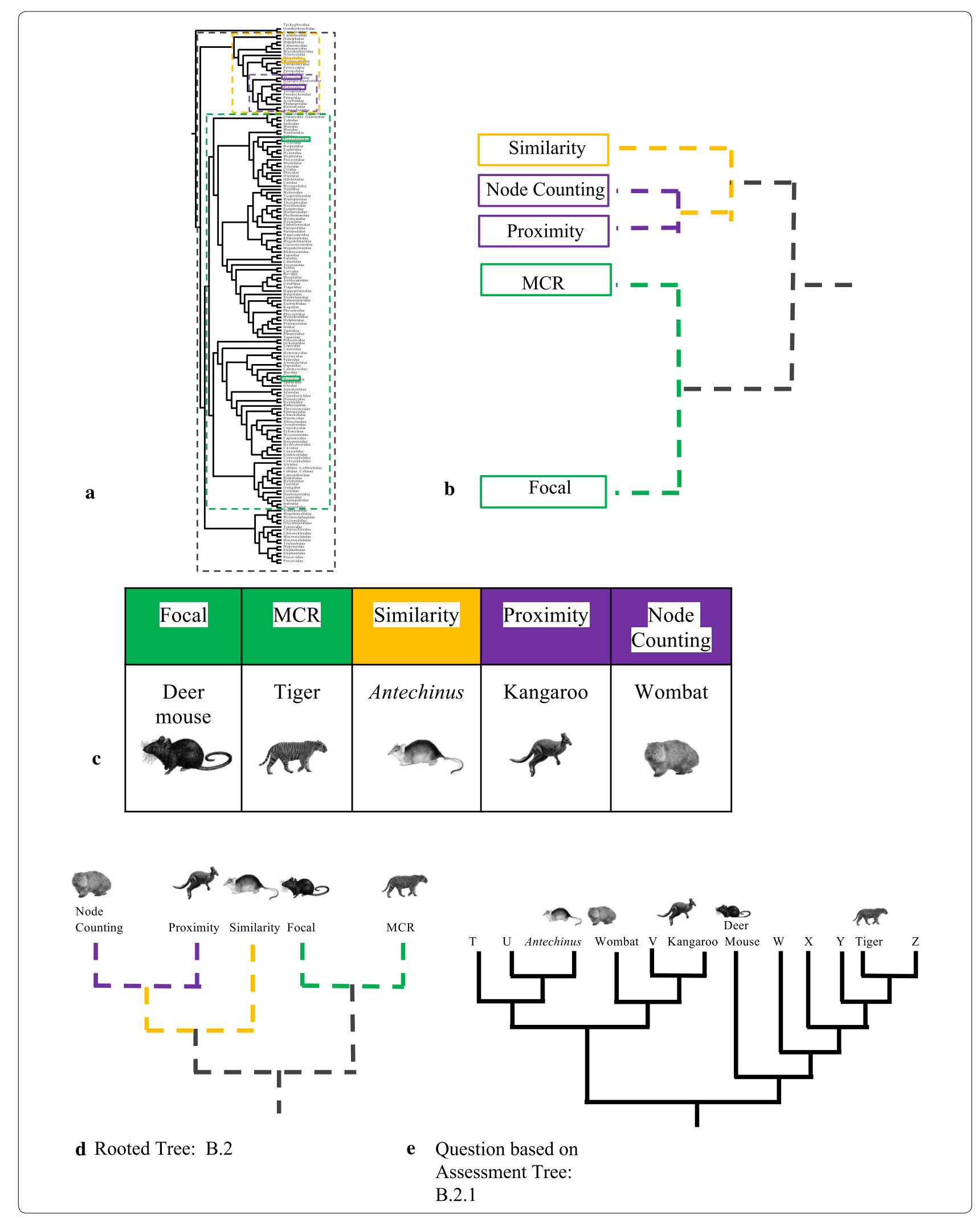




\section{(See figure on next page.)}

Fig. 9 Question development using published phylogeny of arthropods, assessment trees, and images. a Taxa selected from an arthropod phylogeny (modified from Fig. 1 in Meusemann et al. 2010) with clades color-coded. b Assessment categories for each taxonomic group. c Taxa selected for the assessment question: bee (focal; green), ant (MCR; green), hoverfly (similarity; blue), monarch (proximity; blue), and beetle (node counting; yellow). d A color-coded rooted tree, B.6 (Figs. 3, 4b). e Assessment tree B.6.1 (Fig. 4b). The assessment tree is configured by branch addition and clade rotation about nodes so taxa acting as distracters in the question represent their respective incorrect strategy. Sample question: Who is most closely related to the Bee? a. Beetle, b. Hoverfly, c. Monarch, d. Ant

7. Place the taxa and taxa images in the indicated places along the branch tips of the chosen assessment tree.

8. Write the question following the format below, substituting in the taxa names:

Which species is most closely related to the Focal taxon?
a. MCR taxon.
b. Similarity taxon.
c. Proximity taxon.
d. Node counting taxon.

The order of the response choices should vary across questions. One method would be to write the possible responses in the order that they appear from left to right among the tips of the tree.

\section{Example questions developed using published phylogenies}

We show example questions derived from four different phylogenies; each phylogeny was based on a published phylogeny. We use each phylogeny twice to demonstrate how to build separate questions using the same resource. Sample assessment questions are constructed using phylogenies that depict vertebrates (Figs. 5 and 6), mammals (Figs. 7 and 8), arthropods (Figs. 9 and 10), and plants (Figs. 11 and 12). The sample phylogenies may be used to construct binary-choice and four-choice questions.

Figures 5, 6, 7, 8, 9, 10, 11, and 12 contain the following components:

1. Part A: A phylogeny, based on a published phylogeny, depicting the evolutionary relationships of the taxa in the grouping. Color solid-line boxes are shown around the chosen taxa names or their taxonomic classification. Color dashed-line boxes are shown around each clade that the focal taxon shares with each of the other taxa. Color solid and dashed-lines correspond to one another based on the described clades.

2. Part B: A rooted tree depicting the evolutionary relationships among the chosen taxa and the role each taxon will represent in the question: focal, MCR, similarity, proximity, and node counting taxa. Color solid-line boxes are shown around each taxon category. Color dashed-lines show the clade relationships, as described in A.

3. Part C: Images of each taxon including its common name, scientific name, and/or taxonomic classification. Background colors for the taxon category text correspond to the colors in parts a and b in Figs. 5, 6, 7, 8, 9, 10, 11, and 12 .

4. Part D: A color-coded rooted tree with an image of each taxon and their taxon category. Color dashedlines show the clade relationships, as described above.

5. Part E: An example assessment tree with black branches developed from the rooted tree in part D. Assessment trees are uncolored so that they do not display relationship cues in the assessment questions. The correct response to each of the example questions is the first taxon choice that is to the right of the focal taxon on the assessment tree. To eliminate students using this as a cue to answer the question, instead of evaluating evolutionary relationships to determine the correct response, instructors can vary the placement of the correct response. For example, using the mirror image of the example assessment trees. 


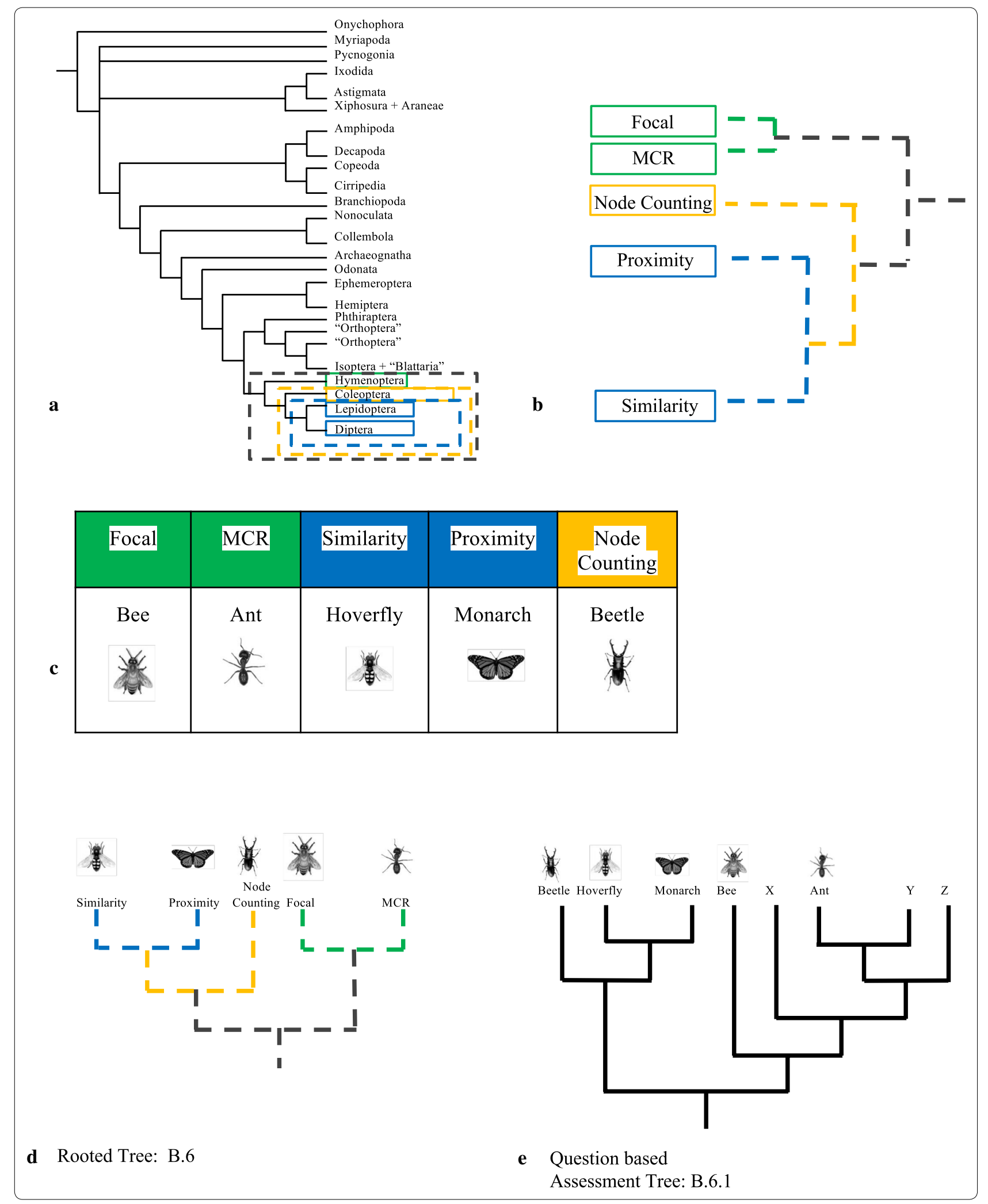


(See figure on next page.)

Fig. 10 Question development using published phylogeny of arthropods, assessment trees, and images. a Taxa selected from an arthropod phylogeny (modified from Fig. 1 in Meusemann et al. 2010) with clades color-coded. b Assessment categories for each taxonomic group. c Taxa selected for the assessment question: ant (focal; green), Daphnia (MCR; green), Myrmarachne (similarity; blue), copepod (proximity; red), and crayfish (node counting; red). d A color-coded rooted tree, C.2 (Figs. 3, 4C). e Assessment tree C.2.2 (Fig. 4C). The assessment tree is configured by branch addition and clade rotation about nodes so taxa acting as distracters in the question represent their respective incorrect strategy. Sample question: Who is most closely related to the Ant? a. Myrmarachne, b. Crayfish, c. Copepod, d. Daphnia

\section{Example of how to design a four-choice question}

The steps for constructing a four-choice assessment question from a published phylogeny focused on vertebrates (Meyer and Zardoya 2003) are shown in Fig. 5. We provide an example of taxa to use, but as mentioned, an instructor designing their own question could use taxa of their choosing. If designing a binary-choice question, simply omit the additional two distracter taxa and responses (Fig. 2).

Example four-choice assessment question construction from vertebrate taxa grouping:

1. Select five taxa whose relationships appear in a published phylogeny (Fig. 5a); each taxon will represent a taxon role (Fig. 5b).

2. Select the focal and similarity taxa: in this case we used the crocodile (focal) and lizard (similarity). The crocodile (focal) is more phenotypically similar to the lizard (similarity) than it is to the chosen MCR, proximity, and node counting taxa (Fig. 5c).

3. Select the MCR taxon: we used the bird (Fig. 5c). The crocodile (focal) shares a more recent common ancestor with the bird (MCR) than it does with the lizard (similarity) and chosen proximity and node counting taxa.

4. Select the proximity and node counting taxa that are not in the clade that contains the crocodile (focal) and the bird (MCR): we used humpback whale (proximity) and bat (node counting); both are eutherian mammals (Fig. 5c).

5. Select the rooted tree from Figs. 3 and 5 that depicts the relationships of the chosen taxa: Fig. 5 uses rooted tree B.1.

6. Place the taxa in the indicated places along the branch tips (Fig. 5d).

7. Select a corresponding assessment tree from Fig. 5b that depicts the relationship from the chosen rooted tree: for rooted tree B.1, we used assessment tree B.1.1.

8. Place letters in place of taxa along the tips that do not have a taxon; letters will not be included as possible answer selections for the multiple choice questions (Fig. 5e).

9. Write the question following the format below, substituting in the taxa names:

Which species is most closely related to the Crocodile?
a. Bird.
b. Lizard.
c. Humpback whale.
d. Bat.

\section{Discussion}

The questions developed using the process described here address understanding of a conceptual model of the evolutionary history of taxa, a phylogeny (phyl: kind or tribe; geny: origin), more specifically, the key tree-thinking skill of determining evolutionary relatedness among taxa on a phylogeny. Given the well-documented problems acquiring this skill, we discuss how tree-thinking questions constructed following our method can be used to improve understanding of evolutionary relatedness among taxa and other evolution concepts.

The method for question construction provides the means for instructors to customize questions in three ways: (1) taxa included, (2) tree structure, and (3) question design. First, instructors may select the taxa of their choice to be included in a question allowing them to design questions with course specificity (e.g., marine organisms for a marine biology course) and select taxa that live in a local ecosystem to increase relevance and student engagement. Freedom in taxa selection also 


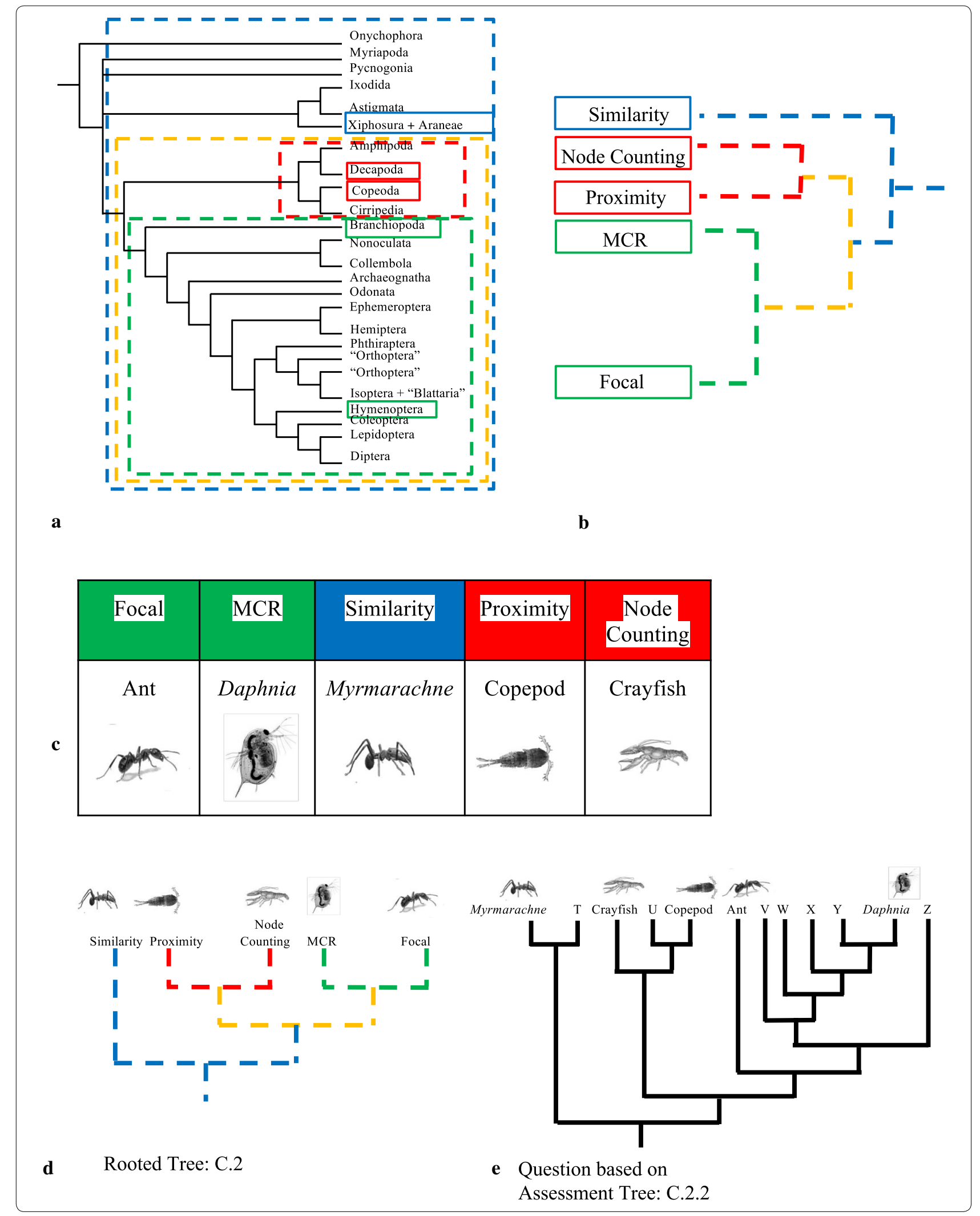


(See figure on next page.)

Fig. 11 Question development using published phylogeny of plants, assessment trees, and images. a Taxa selected from a plant phylogeny (modified from Fig. 1 in Finet et al. 2010) with clades color-coded. b Assessment categories for each taxonomic group. c Taxa selected for the assessment question: palm (focal; green), orange tree (MCR; green), cycad (similarity; yellow), pine (node counting; yellow), and magnolia (proximity; blue). d Color-coded rooted tree B.3 (Figs. 3, 4b). e Assessment tree, B.3.1 (Fig. 4b). The assessment tree is configured by branch addition and clade rotation about nodes so taxa acting as distracters in the question represent their respective incorrect strategy. Sample question: Who is most closely related to the Palm? a. Magnolia, b. Orange, c. Pine, d. Cycad

enables instructors to control question difficulty because they are able to design questions ranging from less difficult (questions containing familiar taxa) to more difficult (questions containing unfamiliar taxa). Next, instructors are able to customize the structure of the evolutionary tree in a question by changing the number of lineages and changing the branching appearance of the lineages. Varying tree structure facilitates question novelty and minimizes false-positive results that arise when students become familiar with a tree structure and are able to answer a question correctly because they recognize a pattern instead of answering correctly because they have acquired the skill of determining evolutionary relatedness. Lastly, instructors can select between two forcedchoice question designs: binary-choice or four-choice enabling instructors to design a question that includes one incorrect strategy and therefore one distracter or includes three incorrect strategies and therefore three distracters.

The method presented here provides a valuable tool for instruction because the question resources are concrete examples that can be used to facilitate discussions about abstract concepts (e.g., homology, homoplasy, synapomorphy, and symplesiomorphy) and commonly held alternative strategies used to interpret evolutionary trees. To exemplify, an instructor can address the incorrect similarity strategy by presenting an assessment tree containing taxa with independent evolution toward a similar body form (homoplasy) as evidence to counter the similarity strategy because parallel selective pressures experienced by the species resulted in similar body form, not common ancestry. Another example of using this method for instruction addresses the proximity incorrect strategy. Constructing assessment trees depicting the same evolutionary relationships among taxa while having different sequences of taxa along the branch tips can be used to demonstrate the fault with the proximity strategy. By presenting phylogenies depicting the same taxa with the same evolutionary relationships but different sequences of taxa along the branch tips, students will be exposed to the inconsequential nature of the sequence of taxa along the branch tips when determining evolutionary relationships among taxa.

Instructors can also use the assessment trees and questions to explore how a phylogeny can show evolutionary relationships among organisms at any taxonomic level. Using phylogenies with a variety of taxonomic levels, instructors can demonstrate to students how clades can be collapsed (lineages extending toward the present from an internal node can be retracted) to represent taxonomic groups that are more inclusive or they can be expanded (lineages can be radiated from terminal taxa) to represent more specific taxonomic groups. When instructors use phylogenies with a diversity of taxonomic groups from less inclusive to more inclusive, greater clarity is given to the properties of internal nodes. Internal nodes represent the hypothetical common ancestors of the lineages; they depict the point at which the taxa in the lineages extending from the internal node exchanged genetic material before reproductive isolation and genetic divergence led to taxa that are recognized as separate from one another. Presenting phylogenies in this way will help dispel the false assumption that evolutionary change only occurs at internals nodes that leads to the alternative strategy node counting.

Questions constructed using the methods presented here can be incorporated within a course as formative 


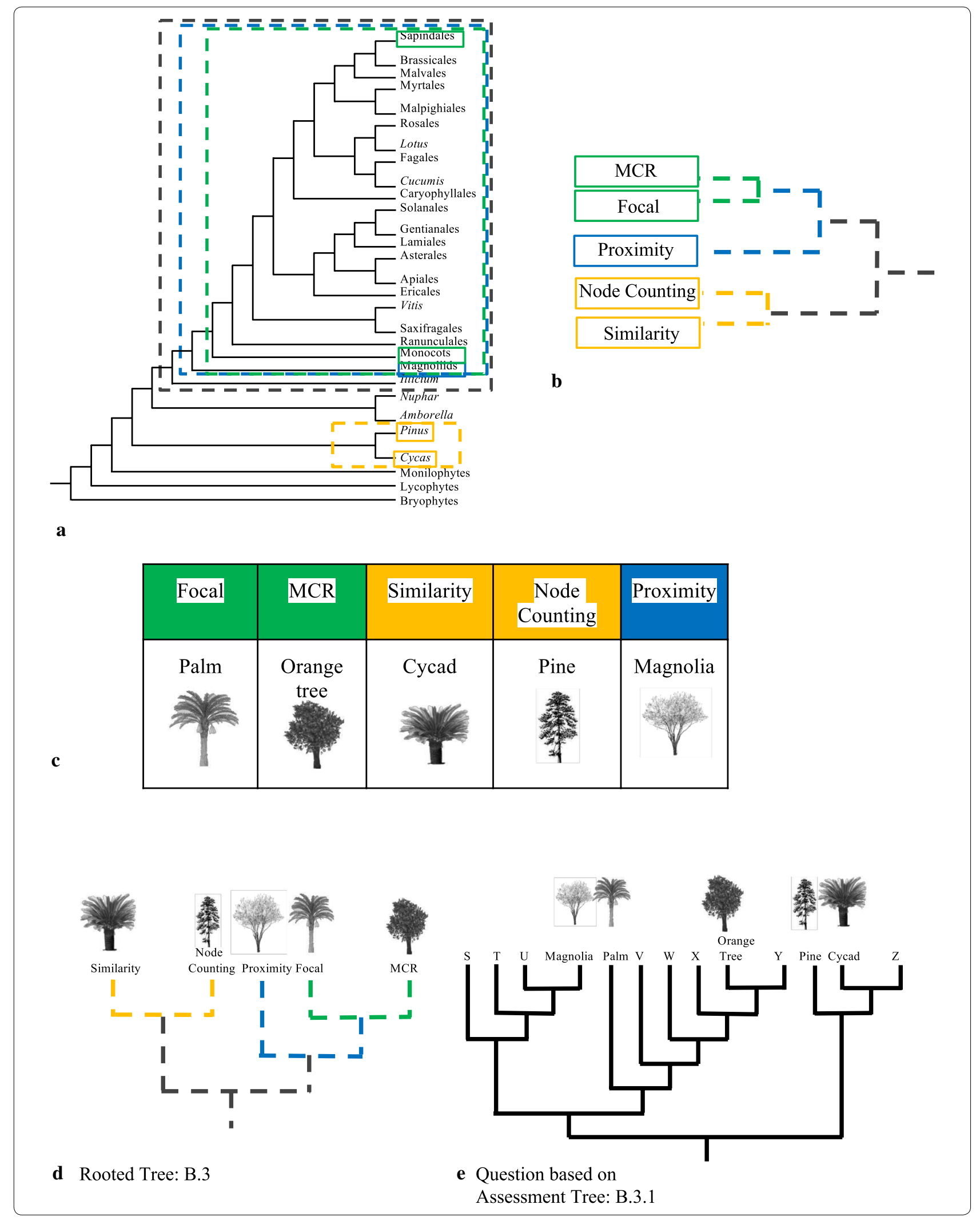




\section{(See figure on next page.)}

Fig. 12 Question development using published phylogeny of plants, assessment trees, and images. a Taxa selected from a plant phylogeny (modified from Fig. 1 in Finet et al. 2010) with clades color-coded. b Assessment categories for each taxonomic group. c Taxa selected for the assessment question: euphorbia (focal; green), cherry tree (MCR; green), saguaro (similarity; purple), sunflower (proximity; blue), and poppy (node counting; yellow). d Color-coded rooted tree, A.1 (Figs. 3, 4a). e Assessment tree, A.1.1 (Fig. 4a). The assessment tree is configured by branch addition and clade rotation about nodes so taxa acting as distracters in the question represent their respective incorrect strategy. Sample question: Who is most closely related to the Euphorbia? a. Poppy, b. Sunflower, c. Cherry, d. Saguaro

and summative assessments. Formative assessments are used within the learning process to provide feedback to the instructor and students about the level of understanding so that deficiencies in understanding can be addressed with further instruction and study (Oosterhof 1999). Therefore, they provide information for metacognition allowing students to monitor and regulate their tree-thinking process. Summative assessments solely focus on how much someone has learned at the completion of a learning cycle (Oosterhof 1999). An example of using questions developed using the method presented here for a summative assessment is an instructor including them on an exam.

The assessment trees and questions developed using the method presented here are a valuable tool in formative assessments because they are intentionally constructed to include common incorrect strategies as distracters to the correct strategy facilitating metacognitive analysis by the students. To obtain an accurate measure of understanding about phylogenies, assessment questions should include the pitfalls that are encountered when interpreting a phylogeny. Including incorrect strategies as distracters can lead to students experiencing cognitive dissonance, the discord of held beliefs with new evidence, if the strategy they employ cannot be used or leads to an incorrect response. Students are motivated to resolve the dissonance (Festinger 1957) therefore eliminating the incorrect strategy and adopting the correct strategy. To further enhance student metacognition and learning, instructors can design learning activities, which include the questions developed using our methods. For example, an instructor could initiate student metacognitive analysis by having students answer questions and also prompt students to explain why the responses they selected as the correct responses are correct and the responses they did not select as the correct responses are incorrect. In addition to students using feedback from the assessment trees and questions to further knowledge acquisition, instructors can use the assessment trees and questions to identify learning deficits and address them with specificity using further instruction or learning activities.

These questions may also be used in a summative assessment to evaluate student understanding at the completion of the learning cycle. If instructors would prefer to not have to create their own evolutionary trees, these assessment trees and example questions are readyto-use. Assessment trees and questions are a valuable resource for summative assessments because they were found to have validity, reliability, and discrimination; in addition, the assessment trees and questions deliberately employ common incorrect strategies as distracters and include taxa commonly seen in college textbooks. 


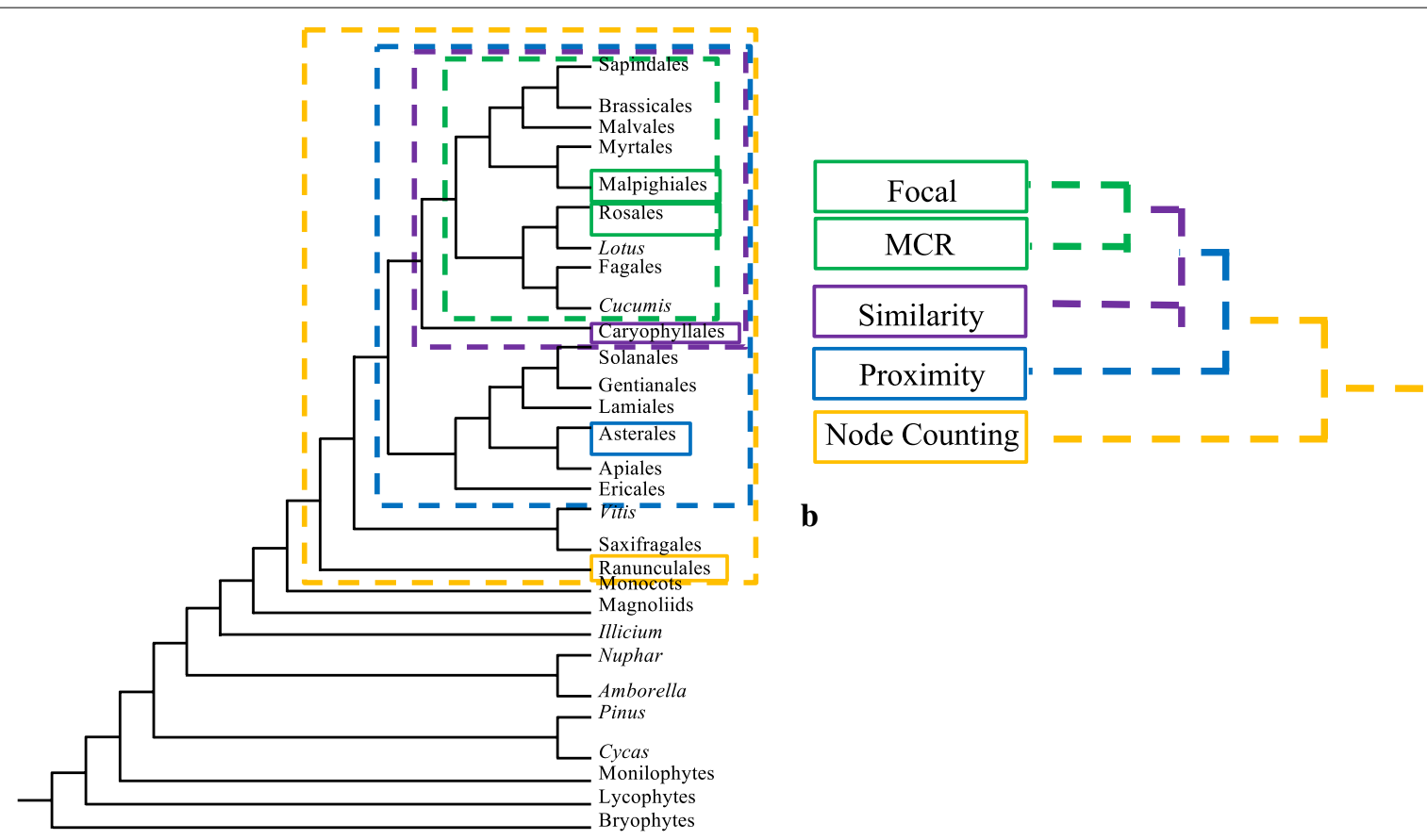

a

\begin{tabular}{|c|c|c|c|c|}
\hline Focal & MCR & Similarity & Proximity & $\begin{array}{c}\text { Node } \\
\text { Counting }\end{array}$ \\
\hline Euphorbia & $\begin{array}{c}\text { Cherry } \\
\text { tree } \\
\text { Poppy }\end{array}$ & Saguaro & Sunflower & Poppy \\
\hline
\end{tabular}
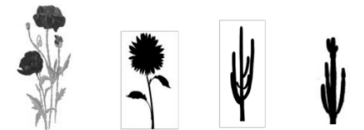

Node

Counting Proximity Similarity Focal

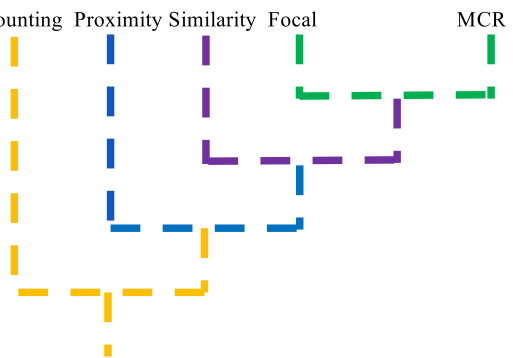

d Rooted Tree: A.1
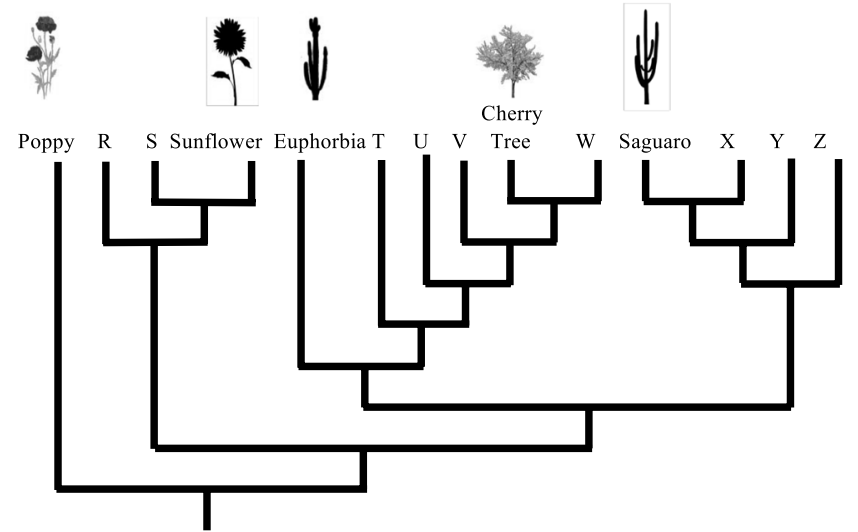

e Question based on Assessment Tree: A.1.1 


\section{Acknowledgements}

Research supported by NSF DUE 0633262 to W. J. Hoese. This project benefited from conversations at the Tree Reasoning in Evolution Education (TREE) workshop at the National Evolutionary Synthesis Center. The authors would also like to thank Jennifer Burnaford, Sean Walker, Shayna Foreman, Asha Mada, Austin Xu, Bryce Renfeldt, Hetal Raval, Tejal Petal, and the other members of the Hoese-Lab.

\section{Authors' contributions}

The authors contributed equally in terms of manuscript development. AAF developed the 4-choice, and LDB developed the binary-choice trees. All authors are personally accountable for their contributions and the accuracy and integrity of the work. All authors read and approved the final manuscript.

\section{Funding}

\section{NSF DUE 0633262 to W. J. Hoese.}

\section{Availability of data and materials}

All data are freely available and materials are provided within the manuscript. The data supporting the conclusions of this article are included within the article and supporting materials.

\section{Ethics approval and consent to participate}

Not applicable.

\section{Consent for publication}

No details, images, or videos relating to an individual person are included in the manuscript.

\section{Competing interests}

Authors have no competing interests.

\section{Author details \\ ${ }^{1}$ Allan Hancock College, 800 South College Drive, Santa Maria, CA 93454, USA. ${ }^{2}$ University of California, Irvine, CA 92697, USA. ${ }^{3}$ California State University, 800 N. State College Blvd, Fullerton, CA 92831, USA.}

Received: 24 June 2020 Accepted: 31 August 2020

Published online: 09 September 2020

\section{References}

Avise JC. Evolutionary pathways in nature: a phylogenetic approach. New York: Cambridge University Press; 2006.

Balding DJ, Bishop M, Cannings C, editors. Handbook of statistical genetics. Chichester: Wiley; 2008.

Baum DA, Smith SD. Tree thinking: an introduction to phylogenetic biology. Greenwood Village: Roberts; 2013.

Baum DA, Smith SD, Donovan SS. The tree-thinking challenge. Science. 2005;310(5750):979-80.

Blacquiere LD, Hoese WJ. A valid assessment of students'skill in determining relationships on evolutionary trees. Evol Educ Outreach. 2016;9(1):5

Catley KM. Darwin's missing link—a novel paradigm for evolution education. Science Education. 2006;90(5):767-83.

Catley KM, Novick LR, Funk DJ. The promise and challenges of introducing tree thinking into evolution education. In: Rosengren K, Evans EM, Brem S, Sinatra G, editors. Evolution challenges: integrating research and practice in teaching and learning about evolution. New York: Oxford University Press; 2012.

Catley KM, Novick LR. Seeing the wood for the trees: an analysis of evolutionary diagrams in biology textbooks. BioScience. 2008;58(10):976-87.

Dees J, Bussard C, Momsen JL. Further effects of phylogenetic tree style on student comprehension in an introductory biology course. CBE Life Sci Educ. 2018;17(2):17.

Dees J, Momsen JL, Niemi J, Montplaisir L. Student interpretations of phylogenetic trees in an introductory biology course. CBE Life Sci Educ. 2014;13(4):666-76.
Fawaz AA. Students' use of alternative strategies when reading evolutionary trees. master's thesis. Fullerton (CA): California State University, Fullerton; 2015.

Festinger L. A theory of cognitive dissonance. Stanford: Stanford University Press; 1957.

Finet C, Timme RE, Delwiche CF, Marlétaz F. Multigene phylogeny of the green lineage reveals the origin and diversification of land plants. Curr Biol. 2010;20(24):2217-22.

Gregory TR. Understanding evolutionary trees. Evol Educ Outreach. 2008; $1(2): 121-37$

Halverson KL. Improving tree-thinking one learnable skill at a time. Evol Educ Outreach. 2011;4(1):95-106.

Halverson KL, Pires CJ, Abell SK. Exploring the complexity of tree thinking expertise in an undergraduate systematics course. Sci Educ. 2011;95(5):794-823.

Kong Y, Thawani A, Anderson T, Pelaez N. A model of the use of evolutionary trees (MUET) to inform K-14 biology education. Am Biol Teach. 2017;79(2):81-90.

Kummer TA, Whipple CJ, Bybee SM, Adams BJ, Jensen JL. Development of an evolutionary tree concept inventory. J Microbiol Biol Educ. 2019;20(2):20.

Meir E, Perry J, Herron JC, Kingsolver J. College students' misconceptions about evolutionary trees. Am Biol Teach. 2007:69(7):71-6.

Meredith RW, Janečka JE, Gatesy J, Ryder OA, Fisher CA, Teeling EC, et al. Impacts of the Cretaceous Terrestrial Revolution and KPg extinction on mammal diversification. Science. 2011;334(6055):521-4.

Morabito NP, Catley KM, Novick LR. Reasoning about evolutionary history: post-secondary students' knowledge of most recent common ancestry and homoplasy. J Biol Educ. 2010;44(4):166-74.

Meusemann K, von Reumont BM, Simon S, Roeding F, Strauss S, Kück P, et al. A phylogenomic approach to resolve the arthropod tree of life. Mol Biol Evol. 2010;27(11):2451-64.

Meyer A, Zardoya R. Recent advances in the (molecular) phylogeny of vertebrates. Annu Rev Ecol Evol Syst. 2003;34(1):311-38.

Novick LR, Catley KM. Understanding phylogenies in biology: the influence of a Gestalt perceptual principle. J Exp Psychol. 2007;13(4):197-223.

Novick LR, Catley KM. Reasoning about evolution's grand patterns: college students' understanding of the tree of life. Am Educ Res J. 2013;50(1):138-77.

Novick LR, Catley KM. Fostering 21st-century evolutionary reasoning: teaching tree thinking to introductory biology students. CBE Life Sci Educ. 2016;15(4):66.

Novick LR, Shade CK, Catley KM. Linear versus branching depictions of evolutionary history: implications for diagram design. Topics Cogn Sci. 2011;3(3):536-59.

Oosterhof A. Developing and using classroom assessments. Prentice-Hall Order Processing Center, PO Box 11071, Des Moines, IA 50336-1071; 1999.

Perry J, Meir E, Herron JC, Maruca S, Stal D. Evaluating two approaches to helping college students understand evolutionary trees through diagramming tasks. CBE. 2008;7(2):193-201.

Phillips BC, Novick LR, Catley KM, Funk DJ. Teaching tree thinking to college students: it's not as easy as you think. Evol Educ Outreach. 2012;5(4):595-602

Sandvik H. Tree thinking cannot taken for granted: challenges for teaching phylogenetics. Theory Biosci. 2008;127(1):45-51.

Schramm T, Schmiemann P. Teleological pitfalls in reading evolutionary trees and ways to avoid them. Evol Educ Outreach. 2019;12(1):1-4.

Smith JJ, Cheruvelil KS, Auvenshine S. Assessment of student learning associated with tree thinking in an undergraduate introductory organismal biology course. CBE-Life Sci Educ. 2013;12(3):542-52.

Starr C, Taggart R, Evers C. Biology: The unity and diversity of life. Australia: Cengage Learning; 2012

Thanukos A. A name by any other tree. Evol Educ Outreach. 2009;2(2):303-9.

\section{Publisher's Note}

Springer Nature remains neutral with regard to jurisdictional claims in published maps and institutional affiliations. 